\title{
Weiter Fortbildung auf dem Zauberberg
}

A

llergien und umweltbedingte Erkrankungen treten immer mehr in den Vordergrund. Neue Erkenntnisse in Diagnostik und Therapie sind deshalb eine wichtige Voraussetzung, um den steigenden Bedürfnissen bei der Behandlung unserer Patienten gerecht zu werden. Den Fortschritt im Bereich der experimentellen und klinischen Immunologie und Allergologie widerzuspiegeln und mitzuhelfen, das neue Wissen unmittelbar in die Praxis umzusetzen, war stets das Anliegen der 1979

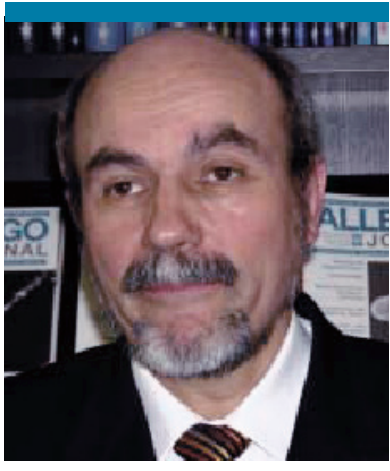

Prof. Dr. Dr. Johannes Ring, Klinik und Poliklinik für Dermatologie und Allergologie am Biederstein, Technische Universität München

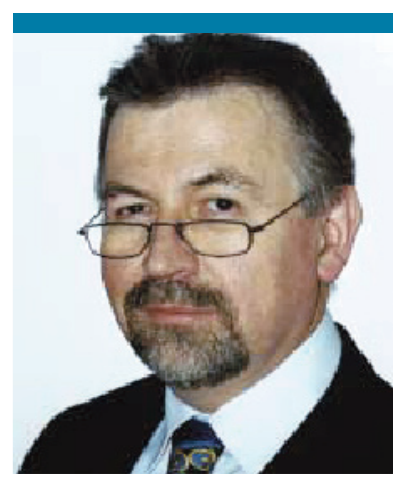

Prof. Dr. Bernhard Przybilla, Klinik und Poliklinik für Dermatologie und Allergologie, Klinikum der Ludwig-MaximiliansUniversität, München von Prof. S. Borelli ins Leben gerufenen Davoser Fortbildungskongresse. Diese Tradition fand im September 2004 mit dem 20. Jubiläumskongress einen vorläufigen Höhepunkt.

Leider kam dann im November 2004 das „Aus“ für die renommierte Alexanderhausklinik - für alle überraschend und bis heute schwer nachvollziehbar. Im gleichen Monat mussten in Davos drei weitere Kliniken schließen, so dass vom „Kliniksterben auf dem Zauberberg" die Rede war. Die Klinik und Poliklinik für Dermatologie und Allergologie am Biederstein der Technischen Universität München hat sich trotzdem entschlossen, die hervorragende Tradition der Fortbildungskongresse in Davos fortzuführen. Diese Kongresse haben ja ihren eigenen Charme und unterscheiden sich von vielen anderen nicht nur durch die vom Klima begünstigte Lage, sondern auch durch die Unkompliziertheit in der Kooperation zwischen verschiedenen Facharztgruppen, Industrie und Gesundheitsbehörden.

Zwischenzeitlich gibt es auch für die Patienten erfreuliche Nachrichten: Durch intensive Verhandlungen ist es gelungen, Verträge mit Rentenversicherungsträgern und Krankenkassen für die Behandlung erwachsener dermatologischer Patienten in der Hochgebirgsklinik Davos-Wolfgang abzuschließen. Die für viele Betroffene so segensreiche Klimatherapie bleibt also nicht nur für Atemwegserkrankungen, sonden auch für Hauterkrankungen bei erwachsenen Patienten weiter möglich. In der schon vorher gemeinsam gegründeten Kinderklinik in DavosWolfgang „Allergie-Klinik - Zentrum für Kinder und Jugendliche" werden seit 2003 Kinder mit allergischen Atemwegsund Hauterkrankungen behandelt.

Bei aller Tradition wurde doch das Konzept des diesjährigen 21. Fortbildungskongresses „Fortschritte der Allergologie, Immunologie und Dermatologie“ geringfügig überarbeitet und stellen-

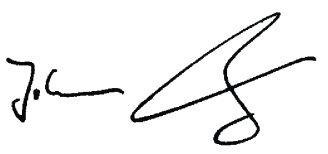

Prof. Dr. Dr. Johannes Ring weise verändert, um den Anforderungen an heutige Fortbildungsveranstaltungen gerecht zu werden. Es wurde deshalb eine besondere Programmauswahl zusammengestellt. Mit Vorträgen, Seminaren und Workshops zu den Themen Asthma, Neurodermitis, Immundefekte, Umweltmedizin, Allergologie, Kinderdermatologie, Andrologie, Mykologie und medizinische Kosmetik hoffen wir, unsere Teilnehmer anzusprechen. Abstracts der Vorträge und Poster finden Sie in dieser Ausgabe von Allergo Journal ab Seite 469.

Weiter geht es dann mit der Antwort auf die Frage, ob Allergien in Deutschland immer noch zunehmen. Sie lautet: Ja! Allerdings hat die Häufigkeit von IgE-vermittelten Sensibilisierungen in der jüngeren Bevölkerung ein Plateau erreicht - wobei unklar bleibt, ob aufgrund der genetischen Ausstattung nicht mehr Menschen sensibilisiert werden können oder ob sich die - letztlich unbekannten - Bedingungen, die zur Epidemie der Allergie führten, geändert haben (Seite 476).

In vielen Fällen kann Allergie durch Hyposensibilisierung geheilt werden. Wie durch sorgfältige Planung weiter entwickelter Protokolle und Analyse der damit erzielten Ergebnisse neue Einsichten gewonnen werden können, lesen Sie ab Seite 482.

Wir wünschen Ihnen eine anregende Lektüre und freuen uns auf ein Treffen in Davos!

Ihre

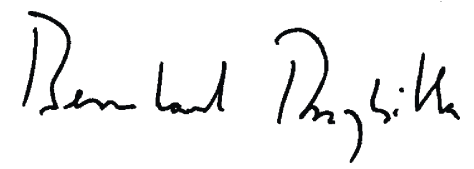

Prof. Dr. Bernhard Przybilla 